\title{
Repurposing of Anthelminthics as Anticancer Drugs
}

\author{
Gerhard Hamilton $\bowtie$ and Barbara Rath \\ Society of Research on the Biology and Treatment of Cancer, A-1160 Vienna, Austria \\ $\triangle$ Corresponding author: Hamilton.SRBTC@gmx.org; Office +43140400-41700 \\ (c) Ivyspring International Publisher. This is an open access article distributed under the terms of the Creative Commons Attribution (CC BY-NC) license \\ (https://creativecommons.org/licenses/by-nc/4.0/). See http://ivyspring.com/terms for full terms and conditions.
}

Received: 2017.04.13; Accepted: 2017.06.25; Published: 2018.01.01

\begin{abstract}
Repurposing refers to the reuse of conventional drugs with distinct indications for new applications in order to speed up drug development by capitalizing on previous knowledge and safety data. A prominent example is the proposal to implement anthelminthics, such as mebendazole, niclosamide and pyrvinium pamoate, as novel anticancer drugs. Numerous studies have demonstrated activity of these agents against a wide variety of cancers, especially cancer stem cell-like subpopulations, by a host of different mechanisms which comprise inhibition of signaling pathways, of mitochondrial respiration, as well as of cellular stress responses and others. However, these anthelminthics were administered orally for the treatment of nematode infections and showed mostly poor resorption and, therefore, systemic toxicity data are frequently not available. Furthermore, the host of different targets described seems to be linked to the capability of the benzimidazoles (mebendazole), salicylanilides (niclosamine) and cyanine dye derivatives (pyrvinium) to interact with DNA directly. In conclusion, anthelminthics poorly fulfill the preconditions of a specific mode of anticancer action and availability of pharmacokinetic and pharmacodynamic data for a favorable repurposing as anticancer drugs.
\end{abstract}

Key words: Anticancer drug, anthelminthics, repurposing, mebendazole, niclosamide, pyrvinium.

\section{Introduction}

Many cancers are still difficult to treat and development of drugs exploiting new pathways and mechanisms involved in tumor initiation and malignant growth is of high priority [1]. However, screening, preclinical testing and clinical trials come at high costs and the whole process is riddled by high attrition rates [2]. An alternative approach to detect new antitumor compounds is the screening of drugs approved for other indications for their efficacy against cancer cells. This so-called process of drug repurposing in oncology may offer several important advantages. Well-characterized drugs may come with detailed knowledge of their pharmacodynamics, pharmacokinetics and side effects as well as with established regimens for clinical applications. In favorable cases, this will result in short-circuiting of the preclinical phase of the drug development $[3,4]$.
Potential agents should possess anticancer activity at physiologically relevant doses, with low toxicity and, preferentially, at low cost [5]. Candidates may reveal another mode of action against cancer cells than in their original indication and require different concentrations to exhibit efficacy in antitumor regimens. Furthermore, they may be cytotoxic or may instead exert an anticancer effect by acting on one or more aspects of the tumor microenvironment either alone or in combination with existing anticancer treatments $[3,4]$.

Examples of drugs identified as high-potential agents within the Repurposing Drugs in Oncology (ReDO) project include mebendazole, cimetidine, nitroglycerin, diclofenac and clarithromycin, among others [3]. Distinct non-cancer drugs already investigated in clinical cancer trials comprise 
metformin, aspirin, hydroxychloroquine and thalidomide [3, 4]. Several drugs investigated for repurposing in oncology are anthelminthics which have been in clinical use against different worms for several decades [6, 7]. Anticancer activities of anthelminthics were reported for mebendazole by Mukhopadhyay et al. in 2002, for pyrvinium pamoate (PPAM) by Esumi et al. in 2004 and for niclosamide by Wang et al. in 2009, respectively [8-10]. Since then, numerous studies have addressed the effects of these compounds against tumor cells in vitro, in vivo and in first clinical studies [3, 4]. Reported anticancer activity of these compounds against tumor-initiating/cancer stem cells (TICs/CSCs) are of special interest in oncology [11]. Besides intracellular signal transduction, a host of other therapeutic targets were discussed for the mediation of the anticancer activity of anthelminthics, ranging from inhibition of mitochondrial respiration, glucose utilization, cellular stress response and others. This review summarizes the current evidence for the anticancer activities of selected anthelminthics and discusses the chemical properties of the compounds as well as new clues regarding their putative targets.

\section{Anthelminthic Drugs}

Helminths are parasitic nematodes that inhabit the human intestine [6, 7]. Anthelminthics were initially developed for treating veterinary parasites and have advanced from treatment of livestock to first clinical applications for patients. These drugs comprise various chemical entities and have several distinct modes of actions. The benzimidazole drugs were reported to bind selectively to $\beta$-tubulin of nematodes, cestodes and fluke, and inhibit microtubule formation and cell division [6]. In detail, agents such as mebendazole and flubendazole induce the loss of cytoplasmic microtubules of the tegumental and intestinal cells of the helminths, and this is followed by loss of transport of secretory vesicles, a decreased glucose uptake and an increased utilization of stored glycogen [12]. The salicylanilides such as rafoxanide, oxyclozanide and closantel and the substituted phenol, nitroxynil, are proton ionophores [6, 7]. Other functionally distinct compounds are classified as nicotinic anthelmintics, glutamate-gated chloride channel potentiators or macrolides.

\section{Mebendazole}

Mebendazole (Methyl N-(5-benzoyl-1H-benzimidazol-2-yl)-carbamate) is a broad-spectrum antihelminthic of the benzimidazole type which came into use in 1971 [6, 7]. It is indicated for the treatment of nematode infestations and the intestinal form of trichinosis. Mebendazole is poorly absorbed into the bloodstream with a bioavailability of $2-10 \%$ [13]. The biological half-life of this drug is 3-6 hours and the metabolism is primarily hepatic with 5-10 \% appearing in urine. Dosing of mebendazole of $30-87$ $\mathrm{mg} / \mathrm{kg} /$ day in humans resulted in plasma levels of $120-218 \mathrm{nM}$ (260 nM for continuous administration) with coefficients of variation ranging from 27 to $72 \%$. Mebendazole can be safely administered to adults and children at high doses over extended time periods. In rare cases, it may result in low white blood cell and platelet count and elevated liver enzymes. Mebendazole is thought to work by interference with microtubules of intestinal cells in parasitic worms, thereby blocking the uptake of glucose and other nutrients, resulting in the immobilization and death of the helminths $[6,13]$.

\section{Niclosamide}

Niclosamide (5-chloro-N-(2-chloro-4-nitrophenyl)-2-hydrobenzamide) is a teniacide which is especially effective against cestodes that infect humans and many animals [14]. Niclosamide displayed toxicity to mammalian cells even at low concentrations [14]. Human volunteers given an oral dose of $2 \mathrm{~g}$ niclosamide exhibited maximal serum concentration of $0.25-6 \mu \mathrm{g} / \mathrm{ml}$ and eliminated the drug within 2 days. The fraction eliminated in urine was up to $25 \%$ and the rest was eliminated with feces. Salicylanilides are very lipophilic and may shuttle protons across membranes, particularly the inner mitochondrial membrane, thus removing the proton gradient and uncouple oxidative phosphorylation [6, $7,15]$.

\section{Pyrvinium}

Pyrvinium is an anthelmintic effective for pinworms [6, 7]. This orange-red compound is poorly soluble in water which hampers its clinical application, except in special formulations. In particular, pamoate is added to pyrvinium to provide a slow release depot form of the drug [16,17]. Pyrvinium pamoate (PPAM) was reported to exert its effects by inhibition of the oxidative metabolism and glucose uptake of the worms [6,7,17]. In 1955, pyrvinium received FDA approval for the treatment of enterobiasis in adults and children (NDA-9582). However, there is no specific assay to measure concentrations of this drug and, following oral administration, it seems to reach the circulation only in very low amounts. The usual human dosage is 5 $\mathrm{mg} / \mathrm{kg} /$ day; however, pyrvinium has been used safely for humans with doses as high as 35 $\mathrm{mg} / \mathrm{kg} /$ day for 3 to 5 days. Accordingly, the drug has no measurable absorption across the gastrointestinal 
tract and $90 \%$ is excreted in feces [18]. At 5 $\mathrm{mg} / \mathrm{kg} /$ day low amounts appeared in liver and plasma in rats.

\section{Anticancer Activities of Mebendazole, Niclosamide and Pyrvinium}

\section{Anticancer activity of mebendazole}

Several studies demonstrated potent antitumor properties of mebendazole (Table 1). The benzamidazole mebendazole significantly inhibited growth of adrenocortical carcinoma cells, both in vitro and in vivo, the effects being due to induction of apoptosis [19]. Moreover, mebendazole inhibited invasion and migration of cancer cells in vitro, and formation of metastases in vivo in experimental animals. Treatment of lung cancer cell lines with mebendazole caused mitotic arrest by depolymerization of tubulin, followed by apoptotic cell death. Oral administration of mebendazole in mice elicited a strong antitumor effect in a subcutaneous model and reduced lesions in experimentally induced lung metastasis without any toxicity when compared with paclitaxel-treated mice $[9,20]$. Furthermore, the drug induced a dose- and time-dependent apoptotic response in chemoresistant melanoma cells via inactivation of Bcl-2 [21]. Mebendazole inhibited melanoma growth with an average $\mathrm{IC}_{50}$ of $0.32 \mu \mathrm{M}$ and preferentially induced apoptosis in melanoma cells compared with melanocytes. Similar antitumor activity of mebendazole was found in gastric cancer, medulloblastoma, glioblastoma, leukemia and myeloma as well as in breast cancer stem cell-like cells [22-26]. Mebendazole potently inhibited hedgehog (Hh) signaling and slowed the growth of Hh-driven human medulloblastoma cells at clinically attainable concentrations [27]. In human cells, mebendazole suppressed the formation of the primary cilium, a microtubule-based organelle that functions as a signaling hub for $\mathrm{Hh}$ pathway activation. Mebendazole can modulate various cancer-associated pathways including ELK1/SRF, AP1, STAT1/2, MEK/ERK, MYC/MAX, dependent on the specific cancer model [28, 29]. Additionally, the drug was shown to inhibit the TRAF2- and NCK-interacting kinase (TNIK), a regulatory component of the $\beta$-catenin and T-cell factor-4 (TCF-4) transcriptional complex downstream of the adenomatous polyposis coli (APC) gene product in colon cancer [30].

Mebendazole at the standard antihelmintic dose of $100 \mathrm{mg}$ twice daily for six weeks stabilized disease in one patient with metastatic colon cancer [27, 31]. Clinical trials employing mebendazole for the treatment of high-grade glioma patients which receive temozolomide and for recurrent/progressive pediatric brain tumors are in the recruiting phase. Furthermore, this drug is tested preclinically as replacement for vincristine for the treatment of brain tumors [32]. Mebendazole can be administered with the non-steroidal anti-inflammatory drug sulindac for prevention of tumor initiation in a colon cancer model [33].

Table 1. Tumor types showing sensitivity to anthelminthics in vitro or animal models

\begin{tabular}{lll}
\hline Mebendazole & Niclosamide & Pyrvinium \\
\hline Colon Cancer & Colon cancer [53], & Colon Cancer [52] \\
{$[28,30,33]$} & $\begin{array}{l}\text { Hepatocellular } \\
\text { Carcinoma [31] }\end{array}$ & \\
& Breast Cancer Triple [29] & Breast Cancer [53] \\
Adrenocortical & Adrenocortical Cancer & \\
Cancer [19] & {$[39]$} & \\
Leukemia/Myeloma & Leukemia/Myeloma [34] & Leukemia/Myeloma \\
[25] & & [59,64] \\
NSCLC [20] & NSCLC [36] & Lung Cancer CSC [57] \\
Cell Lines [9] & Cancer stem cells [46] & Cancer [61] \\
Gastric Cancer [22] & Osteosarcoma [37] & \\
Medulloblastoma [23] & Prostate Cancer [36] & \\
Melanoma [21,29] & Ovarian Cancer/ & Ovarian cancer [62] \\
& TICs [41 - 44] & \\
Breast CSC-like Cells & Breast CSC-like Cells [40] & Breast CSC-like Cells \\
[26] & & [56] \\
Glioblastoma [24,32] & Glioblastoma [33] & Glioblastoma CD133+ \\
& & [60]
\end{tabular}

This table lists reports on investigations employing mebendazole, niclosamide and pyrvinium (pamoate) as anticancer agents against cell lines or in experimental animal models.

\section{Anticancer activity of niclosamide}

A number of studies have established the anticancer activities of niclosamide in both in vitro and in vivo models (Table 1). In combination with cisplatin, niclosamide inhibited epithelialmesenchymal transition (EMT) and tumor growth in triple-negative breast cancer [34]. The cytotoxicity of niclosamide and oxyclozanide were evaluated against HepG2 human liver carcinoma cells and both drugs caused a dose-dependent loss in cell viability $[35,36]$. Furthermore, niclosamide proved cytotoxic for glioblastoma and myelogeneous leukemia stem cells as well as NSCLC cells via NFKB and generation of reactive oxygen species (ROS) [37 - 39]. Inhibition of cell migration and invasion of enzalutamide-resistant prostate cancer cells was effected by niclosamide through its effects on the androgen receptor (AR) STAT3 signaling axis [40]. Growth of osteosarcoma cells was inhibited by this drug by targeting of multiple signal transduction pathways [41]. Furthermore, niclosamide showed significant antitumor activity against a panel of cervical cancer 
cell lines via inhibition of mitochondrial respiration and the mammalian target of rapamycin (mTOR) signaling pathway [42]. In adrenocortical carcinoma, niclosamide has anticancer activity through its inhibition of multiple cellular pathways and impairment of the cellular metabolism [43].

Moreover, the inhibitory effects of niclosamide on cancer stem cells provided further evidence for its consideration as a promising drug for cancer therapy. Niclosamide inhibited proliferation of breast CSC-like cells [44]. Additionally, the drug inhibited growth of experimental ovarian xenografts and the $\mathrm{Wnt} / \beta$-catenin as well as other signaling pathways in ovarian cancer tumor-initiating cells (TICs) [45 - 48]. Furthermore, niclosamide inhibited not only signal transduction, but also targeted mitochondria in cancer cells resulting in cell cycle arrest, growth inhibition and apoptosis [49 - 51]. Niclosamide may work as therapeutic for familial adenomatosis polyposis (FAP) by disrupting the axin-GSK3 interaction and for colon cancer in synergizing with erlotinib [52,53]. Accordingly, clinical trials administrating niclosamide for the treatment of resectable/metastatic colon cancer and castration-resistant, metastatic prostate cancer are recruiting. Among normal tissues, niclosamide showed toxicity against human umbilical vein endothelial cells (HUVECs) and fibroblast-like synovioblasts [54]. Similar results were obtained when the toxicity of niclosamide and oxyclozanide were evaluated against HEK293 human embryonic kidney cells $[35,36]$. In relation to nonmalignant disease, the drug was used to treat an experimental fibrosis in mice [55].

\section{Anticancer activity of pyrvinium pamoate}

The FDA-approved antihelminthic drug, pyrvinium pamoate (PPAM), was found in a screening for compounds that promoted $\beta$-catenin turnover and, thereby, inhibiting Wnt signaling in ovarian and other cancer cells (Table 1) [51, 56]. Furthermore, the drug was characterized as agonist of casein kinases 1 (CK1); however, this effect was not confirmed and in colon cancer cells there was no correlation between PPAM toxicity and mutations in Wnt signaling mediators $[57,58]$. PPAM inhibited mammosphere formation of aggressive breast cancer cells and decreased expression of the CSC surrogate marker aldehyde dehydrogenase (ALDH1) as well as of the epithelial-mesenchymal transition (EMT) phenotype [59]. This drug showed cytotoxicity against colon cancer cells, intestinal polyposis and lymphoma cells via JAK2/STAT5 signaling and impairment of mitochondrial functions [58, 60, 61]. Additionally, PPAM inhibited PI3K-dependent signaling via decreased phosphorylation of both downstream targets of PI3K, AKT and P70S6K [58, 62].

Furthermore, PPAM inhibited lung CSCs derived of cell lines in vitro in the low $\mathrm{nM}$ range [9]. Breast and lung cancer CSCs are targeted by PPAM via Wnt pathway inhibition and medulloblastoma by interference with Hh signaling, respectively [63 - 65]. Furthermore, PPAM induced apoptosis and inhibited self-renewal of stem cells from chemoresistant leukemia patients, while progenitor cells were resistant [66]. Inhibition of the self-renewal in CD133-positive glioblastoma TICs by PPAM was attributed to inhibition of stem cell regulatory pathways [67]. In conclusion, PPAM seems to inhibit TICs/CSCs of various tumor entities via multiple mechanisms with high activity.

The clonal expansion and survival of CSCs seem to be dependent on mitochondrial biogenesis $[8,68$ 71]. Other reported effects of PPAM included inhibition of autophagy which is an important survival mechanism that protects cancer cells against stress, impairment of the unfolded protein response (UPR) induced by glucose starvation and decrease of the activity of STAT3 [72, 73]. We investigated its putative effects on small cell lung cancer (SCLC) circulating tumor cell (CTC) cell lines which are operative in metastasis and exhibit a phenotype overlapping with CSCs [Hamilton, manuscript submitted]. PPAM showed synergism with STAT3 inhibitor S3I-201, glucose hexokinase inhibitor 2-deoxy-D-glucose (2-DG) and dasatinib, thus confirming STAT3 pathway and glycolysis/ autophagy as targets. Out of the 49 RTKs tested, PPAM reduced phosphorylation of 14 kinases, especially of growth factor receptors, proangiogenetic and Wnt mediators. In cytotoxicity assays employing PPAM, we found no significant differences of $\mathrm{IC}_{50}$ values between SCLC tumor cells, SCLC CTC lines which express the Wnt pathway and normal cells such as HEK293 (data not shown). The range of targets of PPAM in cancer cells has not been settled comprehensively.

\section{Therapeutic Targets of Anthelminthics}

The putative targets of the three anthelminthics in cancer cells are summarized in Table 2. For the anticancer activity of mebendazole impairment of the organization of tubulin was identified as main intracellular effect in lung cancer, gastric cancer, leukemia and brain tumors [9, 20, 22, 25, 32]. Cytotoxicity was enhanced by alterations of the regulators of apoptosis, such as Bcl2/XIAP, in melanoma, lung cancer and adrenocortical carcinoma [9, 19, 21, 67]. In human cells, mebendazole suppressed the formation of the primary cilium, a 
microtubule-based organelle that functions as a signaling hub for $\mathrm{Hh}$ pathway activation [27]. Correspondingly, the in vivo growth of hedgehog-dependent medulloblastoma was inhibited by orally administered mebendazole.

Table 2. Anticancer targets of anthelminthics

\begin{tabular}{|c|c|}
\hline \multicolumn{2}{|l|}{$\begin{array}{l}\text { Mebendazole } \\
\text { Targets }\end{array}$} \\
\hline Tubulin & $\begin{array}{l}\text { NSCLC [20], lung cancer [9], gastric carcinoma } \\
{[22] \text {, leukemia [25], brain tumor [32] }}\end{array}$ \\
\hline Apoptosis & $\begin{array}{l}\text { Adrenocortical CA [19], BCL2/XIAP melanoma } \\
{[21,62], \text { BCL-2 lung cancer [9]. }}\end{array}$ \\
\hline Hedgehog pathway & Medulloblastoma [27]. \\
\hline \multicolumn{2}{|l|}{$\begin{array}{l}\text { Niclosamide } \\
\text { Targets }\end{array}$} \\
\hline Signaling pathways & $\begin{array}{l}\text { Cancer cells [45], osteosarcoma [37], STAT3 CSCs } \\
\text { [46], Wnt/ } \beta \text {-catenin ovarian cancer [43], prostate } \\
\text { cancer [36], NSCLC/ c-Jun-ROS [35], colorectal } \\
\text { cancer/TNIK [30]. }\end{array}$ \\
\hline Transcription factors & $\begin{array}{l}\text { Leukemia/NFKB [34], hepatocellular } \\
\text { carcinoma/ATF3 PERK [32]. }\end{array}$ \\
\hline $\begin{array}{l}\text { Androgen receptor } \\
\text { (AR): }\end{array}$ & Prostate cancer [36]. \\
\hline $\begin{array}{l}\text { Mitochondrial } \\
\text { respiration }\end{array}$ & Ovarian cancer [41]. \\
\hline \multicolumn{2}{|l|}{$\begin{array}{l}\text { Pyrvinium } \\
\text { Targets }\end{array}$} \\
\hline Wnt pathway & $\begin{array}{l}\text { Review [50], breast cancer CSCs [56], lung cancer } \\
\text { CSCs [57], ovarian cancer [62]. }\end{array}$ \\
\hline Hedgehog pathway & Medulloblastoma [57]. \\
\hline $\begin{array}{l}\text { Androgen receptor } \\
\text { (AR) }\end{array}$ & Prostate cancer [68]. \\
\hline $\begin{array}{l}\text { Mitochondrial } \\
\text { Respiration }\end{array}$ & $\begin{array}{l}\text { Respiration [62], respiration [63], cancer CSCs } \\
\text { [61], lymphoma [54]. }\end{array}$ \\
\hline Glycolysis & $\begin{array}{l}\text { Glycolysis [8], autophagy [65], UPR/glycolysis } \\
\text { [66] }\end{array}$ \\
\hline
\end{tabular}

A range of different intracellular effects were reported for niclosamide in cancer cells. Signaling pathways were inhibited in osteosarcoma and, especially, Wnt/ $\beta$-catenin signal transduction in ovarian cancer cells and via GSK3 and STAT3 in CSCs and colon cancer $[41,47,50,52,53]$. In prostate cancer cells the STAT3 - androgen receptor (AR) axis was inhibited by niclosamide and cell death in NSCLC was effected via c-JUN stress kinase and generation of ROS $[39,40]$. Transcription factors NFKB and ATF3 were targeted by niclosamide in hepatocellular carcinoma (HCC) and leukemia, respectively $[36,38]$. In ovarian cancer, the effects of the drug were related to the suppression of mitochondrial respiration [45].

Similar to the targets in helminths, pyrvinium impairs glycolysis and mitochondrial respiration in cancer cells. In particular, glucose utilization and related unfolded protein response as well as autophagy were reported to become deranged in diverse cancer cells $[8,72,73]$. Pyrvinium-induced cell death was associated with defective mitochondrial respiration in diverse cancers, breast CSC-like cells and lymphoma, the latter involving regulation of JAK2/STAT5 signaling [60, 68-70]. Several studies reported the Wnt pathway as target of pyrvinium, especially in breast and lung CSCs [56, 63, 64]. Furthermore, the hedgehog pathway, a reactivated embryonic pathway in cancer cells, was reported as pyrvinium-sensitive signal transduction mechanism [65]. Furthermore, pyrvinium demonstrated inhibition of AR activity via selective interaction with a highly conserved DNA-binding domain and was active against castration resistant prostate cancer xenografts [75]. In conclusion, re-investigation of the anthelminthics represents to a major part an on-target repositioning, since impairment of glycolysis, mitochondrial respiration and assembly of microtubules in cancer cells replicates the therapeutic mechanisms reported in nematodes. Inhibition of signaling pathways, especially of the Wnt/ $\beta$-catenin and hedgehog transduction cascades are newly described modalities involved in antitumor activity of the anthelminthics.

\section{DNA and RNA as Therapeutic Targets of Anthelminthics}

The multitude of cellular pathways targeted by anthelminthics is difficult to explain. Almost every mechanism of cancer cells tested was reported to be affected by these drugs, a phenomenon hardly to be traceable back to one or a few underlying molecular targets. The structures of mebendazole, niclosamide and pyrvinium are shown in Figure 1. The common denominator of the three drugs discussed here is their direct interaction with DNA in accordance with their extended molecular structures containing aromatic rings. For pyrvinium we found a typical "light-up" effect upon incubation of this fluorescent compound with cells which indicates an increase of the signal due to intercalation between DNA bases [Hamilton, manuscript submitted].

\section{Benzimidazole}

The binding mode of benzimidazole compounds varies from intercalation to groove-binding, depending on the conformation and size of the compounds [76]. Interest was triggered by the fact that 5,6-dimethylbenzimidazole is a component of vitamin B12 and several antihelminthic, antacid and antibacterial drugs have benzimidazole moieties. Additionally, several benzimidazole-based drugs are well-known for their interaction with DNA and interference with several DNA-associated processes, 
such as topoisomerase II activity and transcription [77]. In addition to the interaction with nucleotidic structures and corresponding processing enzymes, such as topoisomerases, DNA- and RNA-polymerases, benzimidazoles were found to bind to tubulin, disturbing the stability of microtubules [78].

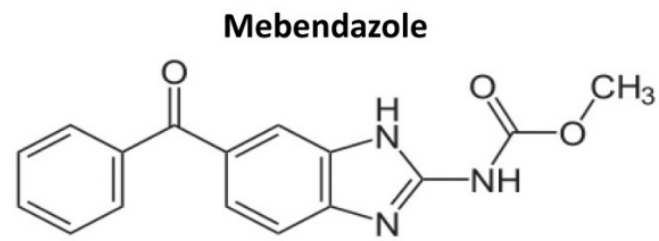<smiles>CN(C)O[Na]</smiles><smiles>Cc1cc(/C=C/c2ccc3cc(N(C)C)ccc3[n+]2C)c(C)n1-c1ccccc1</smiles>

Figure 1. The structures of mebendazole, niclosamide and pyrvinium.

\section{Niclosamide}

The halogenated salicylanilides are a large group of compounds which have been developed mainly due to their antiparasitic activity in animals. The investigation of the niclosamide-DNA interaction using an electrochemical DNA-sensor showed for the first-time clear evidence of DNA binding and suggested that niclosamide toxicity can be caused by this effect, which is observed after reductive activation and transacetylation of the drug [79]. Furthermore, niclosamide was found to activate the DNA damage response (DDR) [80]. Similar results were obtained by electrochemical voltammetric in situ detection of DNA oxidative damage caused by the reduced anthracycline doxorubicin, a classical chemotherapeutic which intercalates into DNA [81].

\section{Pyrvinium}

The substituted quinoline pyrvinium pamoate (PPAM) is a cyanine dye which has been used to treat pinworm (Enterobius vermicularis) infections as well as strongyloidiasis in humans [5, 27]. Pyrvinium exhibits a dark-red fluorescent color which yields a higher signal upon interaction with DNA $[82,83]$. This "light up" effect, the increase of fluorescence intensity upon intracellular binding, is a typical property of DNA intercalators [84, 85]. Although this compound exhibits a preference for AT-rich regions, overall it is expected to affect expression of a multitude of different genes involved in diverse biological functions. For example, PPAM alters the molecular composition of the serotonin receptor $2 \mathrm{C}$ through direct binding to the pre-mRNA causing a conformational change, which makes the regulated splice site more accessible to the splicing machinery, such promoting alternative exon inclusion [86].

\section{Conclusion}

To speed up anticancer drug development, agents in clinical use for nonrelated indications are screened for repurposing or reposition [4]. Approval may be accelerated by knowledge of previous preclinical and clinical data on pharmacokinetics, side effects and regimens [5, 87, 88]. Anticancer activities of several anthelminthics attracted considerable interest, especially their capacity to target signal transduction pathways of CSCs/TICs of several tumor types. Aside of the inhibition of signal transduction, on-target repositioning assumes that the targets recognized in nematods, such as mitochondrial respiration and microtubules, are hit in cancer cells [70, 89, 90]. However, the multitude of effects observed in response to the anthelminthics cannot be explained by specific and defined intracellular targets. Interference with a host of cellular functions tested and synergistic activities with a wide range of other drugs is best explained by direct interaction with various DNA sequences and general interference with replication and transcription, except for some effects exerted by binding to tubulin. In most studies, controls consisting of corresponding non-CSC cells or normal tissues were not included, thus suggesting a non-existing degree of specificity for distinct pathways and unique functions. Furthermore, anthelminthics are administered orally and achieve high concentrations in the gastrointestinal tract but frequently very low concentrations in the circulation. Thus, one of the advantages of repurposing, namely knowledge of previous safety data, would not apply for such drugs in case of systemic application. An exception would be the repurposing of PPAM for the clinical treatment of colonic polyposis [61]. Requirements for the selection of drugs for reposition include evidence of antitumor activity, absence of toxicity in long-term administration and some data of the possible mechanisms of antitumor activity. In 
conclusion, anthelminthics as cancer drugs seem to lack specificity for molecular targets and lack safety profiles for systemic application in large part, such failing to fulfill the basic criteria for successful repurposing in oncology [3]. However, the increasing drug repurposing library for different cancer types may include therapeutics with defined and specific targets for future applications [91].

\section{Acknowledgement}

comments.

\section{Competing Interests}

The authors have declared that no competing interest exists.

\section{References}

1. Buffery D. The 2015 Oncology Drug Pipeline: Innovation Drives the Race to Cure Cancer. Am Health Drug Benefits. 2015; 8: 216-22.

2. Hay M, Thomas DW, Craighead JL, et al. Clinical development success rates for investigational drugs. Nat Biotechnol. 2014; 32: 40-51.

3. Pantziarka P, Bouche G, Meheus L,et al. The Repurposing Drugs in Oncology (ReDO) Project. Ecancermedicalscience. 2014; 8: 442.

4. Ashburn TT, Thor KB. Drug repositioning: identifying and developing new uses for existing drugs. Nat Rev Drug Discov. 2004; 3: 673-83.

5. Bertolini F, Sukhatme VP, Bouche G. Drug repurposing in oncology--patient and health systems opportunities. Nat Rev Clin Oncol. 2015; 12: 732-42.

6. Martin RJ. Modes of action of anthelmintic drugs. Vet J. 1997; 154: 11-34.

7. Hu Y, Ellis BL, Yiu YY, et al. An extensive comparison of the effect of anthelmintic classes on diverse nematodes. PLoS One. 2013; 8: e70702.

8. Esumi H, Lu J, Kurashima Y, et al. Antitumor activity of pyrvinium pamoate,

6-(dimethylamino)-2-[2-(2,5-dimethyl-1-phenyl-1H-pyrrol-3-yl)ethenyl]1-methyl-quinolinium pamoate salt, showing preferential cytotoxicity during glucose starvation. Cancer Sci. 2004; 95: 685-90.

9. Mukhopadhyay T, Sasaki J, Ramesh R, et al. Mebendazole elicits a potent antitumor effect on human cancer cell lines both in vitro and in vivo. Clin Cancer Res. 2002; 8: 2963-9.

10. Wang AM, Ku HH, Liang $\mathrm{YC}$, et al. The autonomous notch signal pathway is activated by baicalin and baicalein but is suppressed by niclosamide in K562 cells. J Cell Biochem. 2009; 106: 682-92.

11. Pantziarka P, Bouche G, Meheus L, et al. Repurposing drugs in your medicine cabinet: untapped opportunities for cancer therapy? Future Oncol. 2015; 11: 181-4.

12. De Nollin S, Van den Bossche H. Biochemical effects of mebendazole on Trichinella spiralis larvae. J Parasitol. 1973; 59: 970-6.

13. Luder PJ, Siffert B, Witassek F, et al. Treatment of hydatid disease with high oral doses of mebendazole. Long-term follow-up of plasma mebendazole levels and drug interactions. Europ J Clin Pharmacol. 1986; 31: 443-8.

14. Rajamuthiah R, Fuchs BB, Conery AL, et al. Repurposing salicylanilide anthelmintic drugs to combat drug resistant Staphylococcus aureus. PLoS One. 2015; 10: e0124595.

15. Andrews P, Thyssen J, Lorke D. The Biology and Toxicology of Molluscidides, Bayluscide. Pharmceut Ther. 1983; 19: 245-95.

16. Beck JW, Saavedra D, Antell GJ, et al. The treatment of pinworm infections in humans (enterobiasis) with pyrvinium chloride and pyrvinium pamoate. Am J Trop Med Hyg. 1959; 8: 349-52.

17. Desai AS. Single-dose treatment of oxyuriasis with pyrvinium embonate. Br Med J 1962; 2: 1583-5.

18. Smith TC, Kinkel AW, Gryczko CM, et al. Absorption of pyrvinium pamoate. Clin Pharmacol Ther. 1976; 19: 802-6.

19. Martarelli D, Pompei $P$, Baldi C, et al. Mebendazole inhibits growth of human adrenocortical carcinoma cell lines implanted in nude mice. Cancer Chemother Pharmacol. 2008; 61: 809-17.

20. Sasaki J, Ramesh R, Chada S, et al. The anthelmintic drug mebendazole induces mitotic arrest and apoptosis by depolymerizing tubulin in non-small cell lung cancer cells. Mol Cancer Ther. 2002; 1: 1201-9.
21. Doudican N, Rodriguez A, Osman I, et al. Mebendazole induces apoptosis via Bcl-2 inactivation in chemoresistant melanoma cells. Mol. Cancer Res. 2008; 6: 1308-15.

22. Pinto LC, Soares BM, Pinheiro J de J, et al. The anthelmintic drug mebendazole inhibits growth, migration and invasion in gastric cancer cell model. Toxicol In Vitro. 2015; 29: 2038-44.

23. Bodhinayake I, Symons M, Boockvar JA. Repurposing mebendazole for the treatment of medulloblastoma. Neurosurgery. 2015; 76: N15-6.

24. Bai RY, Staedtke V, Aprhys CM, et al. Antiparasitic mebendazole shows survival benefit in 2 preclinical models of glioblastoma multiforme. Neuro Oncol. 2011; 13: 974-82.

25. Spagnuolo PA, Hu J, Hurren R, et al. The antihelmintic flubendazole inhibits microtubule function through a mechanism distinct from Vinca alkaloids and displays preclinical activity in leukemia and myeloma. Blood. 2010; 115: 4824-33.

26. Hou ZJ, Luo X, Zhang W, et al. Flubendazole, FDA-approved anthelmintic, targets breast cancer stem-like cells. Oncotarget. 2015; 6: 6326-40.

27. Larsen AR, Bai RY, Chung JH, et al. Repurposing the antihelmintic mebendazole as a hedgehog inhibitor. Mol Cancer Ther. 2015; 14: 3-13.

28. Zhang F, Li Y, Zhang H, Huang E, Gao L, Luo W, et al. Anthelmintic mebendazole enhances cisplatin's effect on suppressing cell proliferation and promotes differentiation of head and neck squamous cell carcinoma (HNSCC). Oncotarget. 2017; 8: 12968-82.

29. Simbulan-Rosenthal CM, Dakshanamurthy S, Gaur A, et al. The repurposed anthelmintic mebendazole in combination with trametinib suppresses refractory NRASQ61K melanoma. Oncotarget. 2017 Feb 21; 8(8): 12576-95.

30. Tan Z, Chen L, Zhang S. Comprehensive modeling and discovery of mebendazole as a novel TRAF2- and NCK-interacting kinase inhibitor. Sci Rep 2016; 6: 33534.

31. Nygren P, Larsson R. Drug repositioning from bench to bedside: tumour remission by the antihelmintic drug mebendazole in refractory metastatic colon cancer. Acta Oncol. 2014; 53: 427-8.

32. De Witt M, Gamble A, Derek Hanson D, et al. Repurposing Mebendazole as a Replacement for Vincristine for the Treatment of Brain Tumors. Mol Med. 2017; 23: 50-56.

33. Williamson T, Bai R-Y, Staedtke V, et al. Mebendazole and a non-steroidal anti-inflammatory combine to reduce tumor initiation in a colon cancer preclinical model. Oncotarget. 2016; 7: 68571-84.

34. Liu I, Chen $X$, Ward $T$, et al. Combined niclosamide with cisplatin inhibits epithelial-mesenchymal transition and tumor growth in cisplatin-resistant triple-negative breast cancer. Tumour Biol. 2016; 37: 9825-35.

35. Di Santo N, Ehrisman J. A functional perspective of nitazoxanide as a potential anticancer drug. Mutat Res. 2014; 768: 16-21.

36. Weng S, Zhou L, Deng Q, et al. Niclosamide induced cell apoptosis via upregulation of ATF3 and activation of PERK in hepatocellular carcinoma cells. BMC Gastroenterol. 2016; 16: 25.

37. Wieland A, Trageser D, Gogolok S, et al. Anticancer effects of niclosamide in human glioblastoma. Clin Cancer Res. 2013; 19: 4124-36.

38. Jin Y, Lu Z, Ding K, et al. Antineoplastic mechanisms of niclosamide in acute myelogenous leukemia stem cells: inactivation of the NF-kappaB pathway and generation of reactive oxygen species. Cancer Res. 2010; 70: 2516-27.

39. Lee SL, Son AR, Ahn J, et al. Niclosamide enhances ROS-mediated cell death through c-Jun activation. Biomed Pharmacother. 2014; 68: 619-24.

40. Liu C, Lou W, Armstrong C, et al. Niclosamide suppresses cell migration and invasion in enzalutamide resistant prostate cancer cells via Stat3-AR axis inhibition. Prostate. 2015; 75: 1341-53.

41. Liao Z, Nan G, Yan Z, et al. The Anthelmintic Drug Niclosamide Inhibits the Proliferative Activity of Human Osteosarcoma Cells by Targeting Multiple Signal Pathways. Curr Cancer Drug Targets. 2015; 15: 726-38.

42. Chen L, Wang L, Shen $\mathrm{H}$, et al. Anthelminthic drug niclosamide sensitizes the responsiveness of cervical cancer cells to paclitaxel via oxidative stress-mediated mTOR inhibition. Biochem Biophy Res Commun. 2017; 484: 416-21.

43. Satoh K, Zhang L, Zhang Y, et al. Identification of Niclosamide as a Novel Anticancer Agent for Adrenocortical Carcinoma. Clin Cancer Res. 2016; 22: 3458-66

44. Wang YC, Chao TK, Chang CC, et al. Drug screening identifies niclosamide as an inhibitor of breast cancer stem-like cells. PLoS One. 2013; 8: e74538.

45. Lin CK, Bai MY, Hu TM, et al. Preclinical evaluation of a nanoformulated antihelminthic, niclosamide, in ovarian cancer. Oncotarget. 2016; 7: 8993-9006.

46. Yo YT, Lin YW, Wang YC, et al. Growth inhibition of ovarian tumor-initiating cells by niclosamide. Mol Cancer Ther. 2012; 11: 1703-12. 
47. Arend RC, Londoño-Joshi AI, Samant RS, et al. Inhibition of Wnt/ $\beta$-catenin pathway by niclosamide: a therapeutic target for ovarian cancer. Gynecol Oncol. 2014; 134: 112-20.

48. Arend RC, Londoño-Joshi AI, Gangrade A, et al. Niclosamide and its analogs are potent inhibitors of Wnt/ $\beta$-catenin, mTOR and STAT3 signaling in ovarian cancer. Oncotarget. 2016; 7: 86803-15.

49. Li Y, Li PK, Roberts MJ, et al. Multi-targeted therapy of cancer by niclosamide: A new application for an old drug. Cancer Lett. 2014; 349: 8-14.

50. Pan JX, Ding K, Wang CY. Niclosamide, an old antihelminthic agent, demonstrates antitumor activity by blocking multiple signaling pathways of cancer stem cells. Chin J Cancer. 2012; 31: 178-84.

51. Carrella D, Manni I, Tumaini B, et al. Computational drugs repositioning identifies inhibitors of oncogenic PI3K/AKT/P70S6K-dependent pathways among FDA-approved compounds. Oncotarget. 2016; 7: 58743.

52. Ahn SY, Kim NH, Lee K, et al. Niclosamide is a potential therapeutic for familial adenomatosis polyposis by disrupting Axin-GSK3 interaction. Oncotarget. 2017; 8: 31842-55.

53. Shi L, Zheng H, Hu W, et al. Niclosamide inhibition of STAT3 synergizes with erlotinib in human colon cancer. Onco Targets Ther. 2017; 10: 1767-76.

54. Huang M, Qiu Q, Zeng S, et al. Niclosamide inhibits the inflammatory and angiogenic activation of human umbilical vein endothelial cells. Inflamm Res. 2015; 64: 1023-32.

55. Murakoshi M, Saiki K, Urayama K, et al. An anthelmintic drug, pyrvinium pamoate, thwarts fibrosis and ameliorates myocardial contractile dysfunction in a mouse model of myocardial infarction. PLoS One. 2013; 8: e79374

56. Ouelaa-Benslama R, Emami S. Pinworm and TNKS inhibitors, an eccentric duo to derail the oncogenic WNT pathway. Clin Res Hepatol Gastroenterol. 2011; 35: 534-8.

57. Venerando A, Girardi C, Ruzzene M, et al. Pyrvinium pamoate does not activate protein kinase CK1, but promotes Akt/PKB down-regulation and GSK3 activation. Biochem J. 2013; 452: 131-7.

58. Wiegering A, Uthe FW, Hüttenrauch $\mathrm{M}$, et al. The impact of pyrvinium pamoate on colon cancer cell viability. Int J Colorectal Dis. 2014; 29: 1189-98.

59. Xu W, Lacerda L, Debeb BG, et al. The antihelmintic drug pyrvinium pamoate targets aggressive breast cancer. PLoS One. 2013; 8: e71508.

60. Xiao M, Zhang L, Zhou Y, et al. Pyrvinium selectively induces apoptosis of lymphoma cells through impairing mitochondrial functions and JAK2/STAT5. Biochem Biophys Res Commun. 2016; 469: 716-22.

61. Li B, Flaveny CA, Giambelli C, et al. Repurposing the FDA-approved pinworm drug pyrvinium as a novel chemotherapeutic agent for intestinal polyposis. PLoS One. 2014; 9: e101969.

62. Zhang $C$, Zhang Z, Zhang $S$, et al. Targeting of Wnt/ $\beta$-Catenin by Anthelmintic Drug Pyrvinium Enhances Sensitivity of Ovarian Cancer Cells to Chemotherapy. Med Sci Monit. 2017; 23: 266-75.

63. Xu L, Zhang L, Hu C, et al. WNT pathway inhibitor pyrvinium pamoate inhibits the self-renewal and metastasis of breast cancer stem cells. Int J Oncol. 2016; 48: 1175-86.

64. Zhang X, Lou Y, Zheng X, et al. Wnt blockers inhibit the proliferation of lung cancer stem cells. Drug Des Devel Ther. 2015; 9: 2399-407.

65. Li B, Fei DL, Flaveny CA, et al. Pyrvinium attenuates Hedgehog signaling downstream of smoothened. Cancer Res. 2014; 74: 4811-21.

66. Xiang W, Cheong JK, Ang SH, et al. Pyrvinium selectively targets blast phase-chronic myeloid leukemia through inhibition of mitochondrial respiration. Oncotarget. 2015; 6: 33769-80.

67. Venugopal C, Hallett R, Vora P, et al. Pyrvinium Targets CD133 in Human Glioblastoma Brain Tumor-Initiating Cells. Clin Cancer Res. 2015; 21: 5324-37.

68. Lamb R, Ozsvari B, Lisanti CL, et al. Antibiotics that target mitochondria effectively eradicate cancer stem cells, across multiple tumor types: treating cancer like an infectious disease. Oncotarget. 2015; 6: 4569-84.

69. Ishii I, Harada Y, Kasahara T. Reprofiling a classical anthelmintic, pyrvinium pamoate, as an anti-cancer drug targeting mitochondrial respiration. Front Oncol. 2012; 2: 137.

70. Tomitsuka E, Kita K, Esumi H. An anticancer agent, pyrvinium pamoate inhibits the NADH-fumarate reductase system-a unique mitochondrial energy metabolism in tumour microenvironments. J Biochem. 2012; 152: 171-83.

71. Harada Y, Ishii I, Hatake K, et al. Pyrvinium pamoate inhibits proliferation of myeloma/erythro-leukemia cells by suppressing mitochondrial respiratory complex I and STAT3. Cancer Lett. 2012; 319: 83-8.

72. Deng L, Lei $\mathrm{Y}$, Liu R, et al. Pyrvinium targets autophagy addiction to promote cancer cell death. Cell Death Dis. 2013; 4: e614.
73. Yu DH, Macdonald J, Liu G, et al. Pyrvinium targets the unfolded protein response to hypoglycemia and its anti-tumor activity is enhanced by combination therapy. PLoS One. 2008; 3: e3951.

74. Doudican NA, Byron SA, Pollock PM, et al. XIAP downregulation accompanies mebendazole growth inhibition in melanoma xenografts. Anticancer Drugs. 2013; 24: 181-8.

75. Lim M, Otto-Duessel M, He M, et al. Ligand-independent and tissue-selective androgen receptor inhibition by pyrvinium. ACS Chem Biol. 2014; 9: 692-702.

76. Kubota $\mathrm{Y}$, Iwamoto $\mathrm{T}$, Seki $\mathrm{T}$. The interaction of benzimidazole compounds with DNA: intercalation and groove binding modes. Nucleic Acids Symp Ser. 1999; 42: 53-4.

77. Bhattacharya S, Chaudhuri P. Medical implications of benzimidazole derivatives as drugs designed for targeting DNA and DNA associated processes. Curr Med Chem. 2008; 15: 1762-77.

78. Lubega GW, Prichard RK. Specific interaction of benzimidazole anthelmintics with tubulin: high-affinity binding and benzimidazole resistance in Haemonchus contortus. Mol Biochem Parasitol. 1990; 38: 221-32.

79. Abreu FC, Goulart MOF, Brett AM. Detection of the damage caused to DNA by niclosamide using an electrochemical DNA-biosensor. Biosens Bioelectron. 2002; 17: 913-9.

80. Ansari J, El-Osta H, Munker R, et al. ATM and ATR protein kinase activity confers cellular resistance to the cytotoxic effects of the anthelminthic drug niclosamide. AACR 2016, abstract 1307.

81. Diculescu VC, Paquim A-MC, Brett AMO. Electrochemical DNA Sensors for Detection of DNA Damage. Sensors. 2005; 5: 377-93.

82. Biver T, De Biasi A, Secco F, et al. Cyanine dyes as intercalating agents: kinetic and thermodynamic studies on the DNA/Cyan40 and DNA/CCyan2 systems. Biophys J. 2005; 89: 374-83.

83. Stockert JC, Trigoso CI, Llorente AR, et al. DNA fluorescence induced by polymethine cation pyrvinium binding. Histochem J. 1991; 23: 548-52.

84. Denny WA. DNA-intercalating ligands as anti-cancer drugs: prospects for future design. Anticancer Drug Des. 1989; 4: 241-63.

85. Neto BAD, Lapis AMA. Recent Developments in the Chemistry of Deoxyribonucleic Acid (DNA) Intercalators: Principles, Design, Synthesis, Applications and Trends. Molecules. 2009; 14: 1725-46.

86. Shen M, Bellaousov S, Hiller M, et al. Pyrvinium pamoate changes alternative splicing of the serotonin receptor $2 \mathrm{C}$ by influencing its RNA structure. Nucleic Acids Res. 2013; 41: 3819-32.

87. Lv J, Shim JS. Existing drugs and their application in drug discovery targeting cancer stem cells. Arch Pharm Res. 2015; 38: 1617-26.

88. Woodsworth DJ, Holt RA. Cell-Based Therapeutics: Making a Faustian Pact with Biology. Trends Mol Med. 2017; 23: 104

89. Würth R, Thellung S, Bajetto A, et al. Drug-repositioning opportunities for cancer therapy: novel molecular targets for known compounds. Drug Discov Today. 2016; 21: 190-9.

90. Lacey E. The role of the cytoskeletal protein, tubulin, in the mode of action and mechanism of drug resistance to benzimidazoles. Int J Parasitol. 1988; 18: 885-936.

91. Chen PC, Liu $X$, Lin $Y$. Drug repurposing in anticancer reagent development. Comb Chem High Throughput Screen. 2016, in press. 